\title{
Love thy neighbor? reciprocal impacts between plant community structure and insect herbivory in CO-occurring Asteraceae
}

\author{
Michael Stastny ${ }^{1,2,3}$ and Anurag A. Agrawal ${ }^{2}$ \\ ${ }^{1}$ Department of Biology, University of Ottawa, Gendron Hall, 30 Marie-Curie, Ottawa, Ontario K1N6N5 Canada \\ ${ }^{2}$ Department of Ecology and Evolutionary Biology, Cornell University, Corson Hall, Ithaca, New York 14853 USA
}

\begin{abstract}
Patterns of herbivory may vary with fine-scale plant community structure: the degree of damage plants experience may depend on their neighbors (i.e., associational resistance or susceptibility). Differential herbivory, in turn, may facilitate a shift in plant community structure. We investigated these reciprocal effects of plant community structure and insect herbivory in a field mesocosm experiment with closely related, native Asteraceae that co-occur in early-successional habitats (old fields). After one year of establishment, we excluded or augmented insect herbivores for two years in equal-density communities of three types: goldenrod-dominated (Solidago spp.) or aster-dominated (Symphyotrichum spp.) congeneric communities and mixtures of the two genera. In manipulated outbreaks, overall and species-specific patterns of herbivory by the main herbivore, the leaf beetle Trirhabda virgata, varied dramatically with community composition. In both years, the preferred goldenrods suffered $25-70 \%$ higher defoliation in mixtures with the less-preferred asters (i.e., associational susceptibility), compared to when growing with congeners; in contrast, asters experienced lower damage in mixtures (i.e., associational resistance). Insect herbivory consistently reduced overall plant productivity, and promoted colonization by other oldfield species. Importantly, herbivory also initiated a shift in the structure of the plant communities, and this effect depended on the starting community composition, implying potential reciprocal effects. For instance, only in mixtures did elevated herbivory reduce the proportional abundance of the preferred host, and the old-field dominant, Solidago altissima. Our findings underscore the importance of plant community composition for variation in and impacts of herbivory and suggest the possibility of feedbacks between herbivory and local community structure as one of the mechanisms contributing to the maintenance of vegetation heterogeneity.
\end{abstract}

Key words: associational resistance; associational susceptibility; community assembly; community structure; competition; dilution effect; herbivory; old fields.

\section{INTRODUCTION}

Herbivores can have important impacts on the structure of plant communities by selectively removing biomass, reducing individual fitness, and altering competitive dynamics among primary producers (Brown and Gange 1992, Coupe and Cahill 2003, Crawley 2009). Conversely, the patterns and intensity of herbivory may be influenced by the quality, quantity, and spatial distribution of their food resource, i.e., the variation in plant community structure (Atsatt and O'Dowd 1976, Agrawal et al. 2006, Cronin et al. 2010, Ness et al. 2011). Feedbacks between these two processes may underlie plant and herbivore dynamics, and contribute to plant coexistence and local maintenance of biodiversity (Carson and Root 2000, Schmitz 2008). Yet, the reciprocal effects between fine-scale plant community structure and the patterns of herbivory have rarely been considered simultaneously in the field (Stein et al. 2010).

Manuscript received 12 June 2013; revised 11 March 2014; accepted 14 March 2014. Corresponding Editor: N. Underwood.

${ }^{3}$ E-mail: mstastn2@uottawa.ca
From an herbivore's perspective, the world comes in many shades of green; plants vary in their suitability as food, having evolved a diverse array of qualitative and quantitative traits to deter or resist attack (Fraenkel 1959, Fritz and Simms 1992, Futuyma and Agrawal 2009). Interspecific and intraspecific differences in defense effectively present herbivores with a fine-scale assortment of plants of differing attractiveness and palatability. The likelihood as well as the extent of herbivory on a given plant species may therefore depend on its neighbors (Agrawal 2004, Agrawal et al. 2006, Barbosa et al. 2009, Ness et al. 2011). This contextdependence of attack is best known as associational resistance: for instance, a highly palatable plant is more likely to escape herbivory when surrounded by less palatable or unsuitable species (Feeny 1976, Hambäck et al. 2000, Barbosa et al. 2009, Hambäck et al. 2014). In contrast, the opposite scenario involves elevated herbivory due to the identity of the neighbors. Associational susceptibility can occur through a variety of mechanisms (e.g., Parker and Root 1981, Karban 1997, Plath et al. 2012), including spillover of herbivores, an induced 
defense response, or depletion of a more palatable neighbor (White and Whitham 2000). In plant communities with co-occurring close relatives, associational effects may be particularly relevant: closely related plant species tend to share herbivores due to their similarity in defensive and nutritional traits (Pearse and Hipp 2009, Ness et al. 2011).

Whether herbivores impose top-down regulation on plant communities that can lead to shifts in community structure has long been a matter of discussion. Herbivory can cause a substantial reduction in plant growth, fitness, and abundance (Schmitz 2008, Crawley 2009), but plant species can also compensate for, or tolerate, damage by herbivores (Trumble et al. 1993). Furthermore, impacts of herbivory often involve complex indirect effects, such as changes in the susceptibility to subsequent herbivory or pathogens (Karban and Baldwin 1997, Daleo et al. 2009), or in competitive ability against other plants (Hambäck and Beckerman 2003). Herbivory may alter the dynamics of plant competition by suppressing species that are competitively dominant (but less resistant or tolerant [Grover 1994, Schadler et al. 2004]), thereby creating opportunities for establishment and proliferation of competitively subordinate species. Through these mechanisms, top-down effects of herbivores may thus lead to increased community evenness and species richness (Carson and Root 2000, Hillebrand et al. 2007).

Given that patterns of herbivory may be influenced by the local plant neighborhood, and damage due to herbivory can alter competitive dynamics among plants, there is a strong potential for interplay between these two processes. However, demonstrating a feedback between them has proven difficult due to the challenges of disentangling these mutually dependent effects. Modeling approaches and long-term demographic data have been used to study density dependence and population dynamics of plants and herbivores, and typically are quantitatively or experimentally intensive, even in studies that focus on single plant species (Doak 2000, Underwood and Halpern 2012). Much less attention has been given to these topics in a multispecies community context, in which substantial variation in reciprocal plant-herbivore interactions, including associational effects, could be expected in multiple host or non-host species simultaneously. An investigation of the mechanisms through which plant community structure and insect herbivory could be mutually linked represents a first step toward our improved understanding of the maintenance of plant and herbivore diversity at local scales.

We use a novel approach to begin to disentangle reciprocal effects of plant community structure and insect herbivory in a multiyear, field mesocosm experiment. We chose seven closely related, perennial goldenrods and asters (Asteraceae; tribe Asterae) that dominate early successional "old fields" in central New York state (USA). These species naturally co-occur but vary in abundance and in rates of herbivory (Stastny 2010), and share a rich assemblage of phytophagous insects, including several species with periodic multiyear outbreaks (Maddox and Root 1990, Root 1996). Context-dependent herbivory may thus contribute to the outcomes of plant competition and mediate local coexistence of these close relatives. To address this putative link between the variation in plant community structure and insect herbivory, our study tested whether changes in community composition can be explained, in part, by associational effects of herbivory. To do this, we planted two types of "congeneric," communities, dominated by goldenrod or aster congeners, respectively, and mixtures of the genera. After a year of herbivore exclusion to facilitate plant establishment, we exposed half of the mesocosms to insect herbivory for two consecutive growing seasons, including two manipulated outbreaks of the key herbivore, Trirhabda virgata (Chrysomelidae).

Our investigation of the effects of plant community structure on the variation in, and consequences of, insect herbivory was motivated by the following questions. First, do patterns of herbivory among these related host plants vary with community composition? Second, does context-dependent herbivory affect relative species performance, contributing to a shift in plant community structure and assembly? Demonstrating such reciprocal impacts between community structure and herbivory is a first step toward understanding the dynamics and regulation of ecological feedbacks between them.

\section{Methods \\ Study system}

The herbaceous successional habitats ("old fields") of northeastern North America are dominated by several genera of native Asteraceae, which support a rich fauna of specialist and oligophagous insect herbivores. We selected two locally abundant genera, Solidago (goldenrods) and Symphyotrichum (New World asters; hereafter, asters), and represented each by three of the most common species that frequently co-occur even at the scale of microsites (M. Stastny, unpublished data): Solidago altissima, So. juncea, and So. rugosa, and Symphyotrichum lateriflorum, Sy. pilosum, and $S y$. urophyllum. In all mesocosms, we also included Euthamia graminifolia, another common and co-occurring species that is ecologically similar to Solidago (and formerly in this genus), to address a different question described elsewhere (Stastny 2010).

The key insect herbivore in our study, Trirhabda virgata (hereafter, Trirhabda), is a univoltine Chrysomelid leaf beetle whose larvae and adults consume the foliage of goldenrods and asters. Known primarily as an outbreaking specialist on Solidago altissima (e.g., Root and Cappuccino 1992), it naturally feeds on all seven species in our study, even when the primary host is available (Stastny 2010). However, the larvae and especially the adults display a strong preference for So. 
altissima and other goldenrods over asters, although larval performance is similar among the hosts (Stastny 2010). The mobile larvae are the most damaging life stage; during localized outbreaks (May to early June), their densities of up to 30 per stem can lead to complete defoliation (M. Stastny, personal observation). The adults feed until early autumn, but at densities lower by several orders of magnitude, likely due to postemergence dispersal (Herzig 1995).

The study was conducted between 2007 and 2009 (years 1, 2, and 3, hereafter) in an old field near Ithaca,

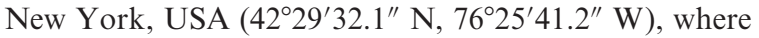
all seven focal species co-occur. Abandoned from agricultural use over 40 years ago (R. B. Root, personal communication), the field is adjacent to a site where studies on herbivory and plant community dynamics have been conducted since 1970s (Whipple Farm; Carson and Root 2000). Dominated by native, perennial Asteraceae, it is representative of old fields in the region.

Plants were grown from seed collected from 20-40 individuals per species in nearby old fields, selected at least $15 \mathrm{~m}$ apart to avoid repeated sampling from the same clones. In April 2007, seeds from 15-25 maternal plants per species with sufficient germination rates were pooled, and germinated in clusters on moist soil surface (Metro Mix; SunGro Horticulture, Agawam, Massachusetts, USA) in 72-cell trays in a greenhouse, at a $14 \mathrm{~h}$ light : $10 \mathrm{~h}$ day photoperiod. The seedlings were thinned randomly as needed to ensure one plant per cell. The trays were periodically reshuffled to minimize position effects. After five weeks, the plants were acclimated in outdoor cages for several days before transplanting. Seedlings of similar size were selected for each mesocosm in order to reduce size-dependent variation in initial competitive interactions.

The circular mesocosms were constructed to maximize realism of field conditions while effectively confining the plant communities. In May 2007, 120 holes $(90 \mathrm{~cm}$ diameter, approximately $30 \mathrm{~cm}$ deep) were drilled with a mechanical auger, spaced out in an area of $200 \times 40 \mathrm{~m}$ to capture the natural variation in soil moisture, nutrients, and other abiotic conditions. A strip of aluminum flashing was inserted vertically along the walls of each hole, and all field soil was returned into it. The flashing thus created a barrier around the perimeter of each mesocosm, extending 22-27 cm deep and 3-6 cm above the soil surface, which contained each experimental community and prevented encroachment of vegetation belowground. Each mesocosm was surrounded by a wire fence (approximately $2 \times 2 \mathrm{~m}, 1.3 \mathrm{~m}$ height) to exclude deer and rabbit browsing.

\section{Composition treatment}

In a randomized block design with four blocks $(40 \times$ $50 \mathrm{~m}$ ), we assigned all mesocosms to one of three types of initial composition ( $n=40$ mesocosms at each level): a congeneric community of goldenrods, a congeneric community of asters, and a confamilial mixture of the two genera (hereafter, mixtures). The congeneric mesocosms contained three species from the respective genus, with six individuals per species. The mixtures contained all six species (i.e., three Solidago species and three Symphyotrichum species), with three individuals per species. In all communities, we also included three individuals of Euthamia graminifolia, to address a separate question presented elsewhere (Stastny 2010), at the constant proportion of one-seventh of the community. Hence, all mesocosms started at an equal initial density of 21 genets, planted in a substitutive (replacement) design, and at species richness of either four species in the congeneric communities (i.e., congeners plus Euthamia), or all seven species at equal proportions in the mixtures. Note that nearly all of the changes in the relative abundance of the focal species thereafter were driven by the subsequent variation in the biomass of the initial 21 long-lived genets, through increased size and stem number, and clonal growth via rhizomes (goldenrods and Euthamia). Therefore, species relative abundances in year 3 reflect variation in performance rather than demographic changes; no new individuals (genets) of the focal species were recruited except, to a very limited extent, seedlings.

The initial 21 genets were planted at even spacing while avoiding clumping of conspecifics as much as possible. For several weeks, the mesocosms were watered periodically to aid establishment. All 2520 plants survived the first growing season, and in the following two years fewer than $0.5 \%$ of the original genets were lost due to mortality. During year 1, the mesocosms were weeded to prevent disturbance-associated ruderals from outgrowing the focal species. All subsequent plant recruitment or encroachment (hereafter, "colonization") took place without any intervention, allowing us to test whether initial composition and herbivory interacted to produce differential patterns of colonization by other old-field species.

\section{Herbivory treatment}

In year 1, all 120 mesocosms were protected from insect herbivory with a non-systemic insecticide (esfenvalerate, as Bug-B-Gon, Ortho; Scotts Miracle-Gro, Marysville, Ohio, USA) once every other week during the peak growing season (mid May to early October). Esfenvalerate and fenvalerate have been used previously in this and other systems, and shown to effectively reduce insect herbivory without phytotoxic effects on plants, and with negligible impacts on soil processes and fertility (Mitchell 2003, and references therein). In year 2, we assigned each of the mesocosms to either insect herbivory or insect exclusion. These treatments were fully crossed with the three community composition levels in the randomized block design, yielding 20 replicates per treatment combination. Insect exclusion involved continuing the insecticide application. At each application, the mesocosms in the other treatment were sprayed with an equal amount of water. 
The insect herbivory treatment relied on supplementing the ambient levels of damage; hence, there is no treatment that represents natural herbivory levels. Instead, we created consecutive outbreaks of Trirhabda in year 2 and year 3 by releasing larvae into the mesocosms. Each year in late May, about 20000 larvae of mixed ages (with the majority in the second or third instar) were collected from an outbreaking population in a nearby old field. An equal number of larvae (about 400) was then evenly distributed ("sprinkled") throughout each of the 60 mesocosms, which, by that point, contained 60-150 ramets. This density approximates median per-ramet densities during local Trirhabda outbreaks (M. Stastny, personal observation); however, the actual per-ramet loads effectively became lower in mesocosms with a lower proportion of the preferred hosts (i.e., goldenrod), as the mobile larvae could emigrate into mowed buffers and forage beyond. The larvae fed in the mesocosms for 8-15 days prior to pupation.

In addition to Trirhabda outbreaks, mesocosms open to insect herbivory also experienced feeding by a rich assemblage of other insect herbivores, including leaf rollers, galling insects, spittle bugs, flea beetles, and others (see Maddox and Root 1990). The densities of these herbivores were not manipulated, and were too low for statistical inference. Trirhabda adults continued feeding in the herbivory treatment; however, their densities were at least two orders of magnitude lower than those of the larvae (M. Stastny, personal observation), precluding quantitative comparisons among the host species and composition treatments.

\section{Data collection}

Immediately following the outbreak, and before plant growth could confound our relative measures of defoliation, we surveyed foliar damage in each mesocosm by randomly selecting and averaging eight ramets per species. In the small minority of cases when fewer than eight ramets were available, all extant ramets were included. Damage was estimated visually as the percentage of leaf area removed by larval feeding, to the closest $5 \%$. This unweighted, plant-centric metric reflects the relative rather than absolute level of defoliation for each species, allowing a more meaningful inference about the impacts of herbivory on relative plant abundance. Nearly all stems received at least some feeding damage, indicating that the larvae sampled among the different hosts. We conducted the same survey in the insecticide treatment to confirm the efficacy of the insect exclusion treatment. In September 2008, when insect herbivores began to decline and the plants reached their full vegetative size, we re-surveyed foliar damage following the same procedure as above. This survey represents cumulative herbivory by a variety of chewing insect herbivores (see Maddox and Root 1990), including Trirhabda, but also incorporates any post- outbreak growth; the results are presented in Appendix A: Fig. A1.

Every fall, we harvested the aboveground biomass of each species in individual mesocosms only once most of its aboveground parts had senesced (late October to early November), so as not to affect the belowground allocation for regrowth, given among-species variation in phenology. All biomass was dried for 48 hours at $60^{\circ} \mathrm{C}$ and weighed. In July 2009 , we conducted a survey of plants that had colonized the experimental communities after the first year. We identified and recorded all the species ("colonizing species"), and estimated their individual percent cover. In order to accurately capture the vertical complexity of the vegetation, as colonization occurred primarily in the understory of the dominant focal species, our method allowed for counting the same area two or more times if occupied by two or more overlapping species; i.e., the percent cover for all species could sum to over $100 \%$. Because of the late harvest, and to avoid earlier disturbance, we were unable to measure the biomass of the colonizing species, many of which had senesced much earlier.

\section{Statistical analysis}

All of the analyses involved linear models or linear mixed effects models, using $\mathrm{R}$ software (version 2.15.2; $\mathrm{R}$ Development Core Team 2013). Block was not included in any of the analyses; in preliminary mixed effects models (function lmer) with block as a fixed effect (due to its low number of levels [Crawley 2002]), block and its interactions were never significant (log likelihood ratio test [Bolker 2008]). Whenever a response variable was measured over multiple years, year was included as a fixed effect and mesocosm as a random effect in the repeated measures linear mixed model to avoid pseudoreplication. In all univariate analyses of variance (or covariance), we used the information theoretic (Akaike's information criterion, AIC) approach in stepwise model selection, and Markov chain Monte Carlo (MCMC) sampling (function pvals.fnc) to estimate $P$ values for the fixed effects in the mixed effects models.

Where community-level response variables were tested, we included composition as a fixed effect with three levels (congeneric goldenrods, congeneric asters, and mixtures). In analyses focusing on species-level response variables, we instead used composition as a fixed effect with two levels (congeneric, mixture) in combination with a fixed effect of genus (goldenrods, asters). This parameterization accurately accounts for our specific replacement design, in which congeneric communities were either goldenrod or aster dominated, and only mixtures contained all seven species. Furthermore, it allows us to independently examine the responses of goldenrods (the preferred hosts) vs. asters to different ecological contexts by including the relevant interactions of genus and treatment(s) of interest. Species and mesocosm were included as random effects, 
enabling us to model the covariances among the species within individual mesocosms (units of replication).

In the species-level analyses involving the effect of genus, we grouped Euthamia (present in all mesocosms) with its original taxonomic grouping, the goldenrods, as they share many ecological similarities in vegetative traits and in herbivores (Stastny 2010); however, we found similar results when we excluded it. For parsimony, improved model performance, and consistency among our analyses, we present the former approach.

Effects of community context on herbivory.-To test the effect of the initial community composition on herbivory by Trirhabda larvae, we analyzed feeding damage using two approaches. The first approach focused on herbivory at the community level, using the percent defoliation averaged across all focal species in each mesocosm (i.e., mesocosm means). We used a linear mixed model (lmer) with the initial composition (three levels) and year (2 and 3 ) as the main effects (plus their interaction), and two a priori orthogonal contrasts (Bolker 2008). The first contrast compared herbivory between the congeneric communities of goldenrods and asters; the second contrast tested whether the mixtures experienced lower (or higher) herbivory than would be expected given the levels in the congeneric communities (i.e., whether the mixtures differed from the congeneric mean), suggesting nonadditive (and potentially associational) effects.

In the second approach, we tested for associational effects when damage on individual species and genera was considered. Specifically, we examined whether initial community composition influenced herbivory, and whether this composition effect on damage differed between goldenrods and asters. We used a linear mixed model (lmer), with composition (congeneric, mixture), genus (goldenrods, asters), and year as fixed effects, and species and mesocosm as random effects. The response variable (damage) was log-transformed to meet parametric assumptions. A significant effect of composition or genus would indicate that, overall, individual species received different levels of damage between congeneric communities and mixtures, or depending on their genus, respectively. A significant composition $\times$ genus interaction would indicate that the effect of community context on damage differed between goldenrods and asters.

Impacts of herbivory on community structure.-

1. Primary productivity.-We tested the overall impact of herbivores on the plant communities by comparing community productivity (total aboveground biomass of the focal species) between the herbivory treatments. Although mesocosm-level response variables were not correlated between years 2 and 3, we included year in the linear mixed model (lmer), in addition to the main effects of herbivory (presence/absence), composition (three levels), and their interaction. The test employed two a priori orthogonal contrasts of composition treatments (see Effects of community context on herbivory). A significant herbivory $\times$ composition interaction would indicate that the impacts of herbivory on productivity depended on the community context.

2. Community assembly.-The effect of herbivory on the colonization of the mesocosms by other plant species, and its contingency on the initial community structure, were analyzed in a linear model (function $\mathrm{lm}$ ) that included herbivory (presence/absence) and composition (three levels) as the main effects, and their interaction. The two dependent variables, species richness of colonizing species and their total percent cover, were log-transformed to meet parametric assumptions and analyzed separately. We employed the same a priori orthogonal contrasts involving composition (see Effects of community context on herbivory). Community productivity, a potential determinant in colonization, was initially included as a (nonsignificant) covariate but dropped from the model.

3. Relative abundance.-We assessed the impact of insect herbivores on plant community structure after two years by testing the overall effect of the herbivory treatment on the individual species abundances, and whether this effect varied with initial community composition and genus. We used standardized proportional biomass of each species in the final year as the dependent variable (log-transformed to meet parametric assumptions), to represent its relative abundance in the community. This metric was calculated by dividing the aboveground biomass of each species in the mesocosm by its initial number of genets, to allow a comparison of the congeneric communities and mixtures (six and three genets, respectively), and then dividing this value by the total community biomass in the final year. We employed a similar mixed model framework (lmer) as in the species-level analysis of damage (but without the year effect), with species and mesocosm as random effects. The model included the fixed effects of composition (congeneric, mixtures) and herbivory (presence/absence), and their interaction, as well as their respective interactions with genus (goldenrods, asters), and the three-way composition $\times$ herbivory $\times$ genus interaction. To account for the variation in species biomass before the herbivory treatment began in year 2, initial proportional biomass (year 1) was included as a covariate. A significant interaction between composition and herbivory would indicate that the impact of herbivory on species relative abundance by year 3 depended on initial community structure; a significant three-way interaction with genus would indicate that the composition-dependent effect of herbivory differed between goldenrods and asters.

For a closer examination of the interplay between community context and herbivory, we next focused on So. altissima, the dominant species in old fields and preferred host of Trirhabda. First, using a linear mixed model (lmer), we evaluated the impact of herbivory and community composition on the relative abundance of So. altissima across years 2 and 3 . We used its standardized proportional biomass as the dependent 


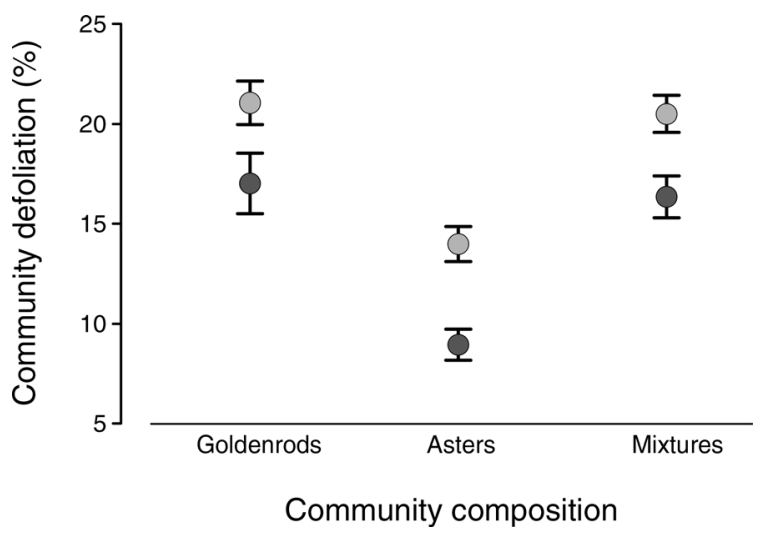

FIG. 1. Community defoliation (percentage of total leaf area consumed) by Trirhabda virgata larvae after manipulated outbreaks (herbivory treatment only) in year 2 (dark gray circles) and 3 (light gray circles), comparing across types of community composition: congeneric goldenrod communities, congeneric aster communities, and the mixtures of the two genera. Values are means $\pm \mathrm{SE} ; n=20$ mesocosms per treatment.

variable, and included community productivity as a covariate to account for environmental variation among mesocosms. Based on stepwise model selection (Bolker 2008), the final model included the fixed terms of composition, herbivory, year, as well as the two-way and three-way interactions. A significant composition $\times$ herbivory interaction would indicate that the impact of herbivory on So. altissima abundance depended on its community context.

To complement the analysis, which tested whether the slopes of the relationship between herbivory (presence/ absence) and So. altissima abundance varied with community composition, we employed a regression approach with herbivory as a continuous variable (i.e., damage, in the presence of herbivory only) to test if both of those slopes differed from zero. We ran two multiple linear regressions (linear model, function $1 \mathrm{~m}$ ), one for goldenrod communities, the other for mixtures, to test whether the relative abundance of this species in the final year was predicted by damage in year 2 and 3 , and by its previous abundance. We then compared the relative importance of these predictors between the two models (composition treatments). Proportional biomass in year 3 was the dependent variable; damage in year 2 and year 3 , and proportional biomass in year 2 (but not in year 1 , due to its colinearity with biomass in year 2), were the independent covariates; all variables were first standardized (converted to $Z$ scores, function scale in $\mathrm{R}$ ). We used the standardized regression coefficients to infer the relative importance of the predictor variables between the two composition types.

\section{Results}

Effects of community context on herbivory.-Community composition was an important determinant of herbivory. Congeneric communities of goldenrods re- ceived, overall, significantly more defoliation by Trirhabda larvae than those of asters (Fig. 1): their average percent leaf area removed was $90 \%$ and $51 \%$ higher in year 2 and 3, respectively (overall difference; Appendix B: Table B1; composition effect, $P<0.001$ ). However, in both years, the mixtures consistently experienced overall defoliation comparable to that of goldenrod communities (Fig. 1), i.e., significantly greater than would be expected given the levels of herbivory in the two congeneric communities (contrast of the mean congeneric communities and mixtures; Table $\mathrm{B} 1 ; P=$ 0.019). Therefore, at least some species consistently received more damage in the mixtures than in their respective congeneric communities.

In both outbreak years, defoliation by Trirhabda at the species level revealed a contrasting pattern of associational resistance vs. susceptibility between the community types, and this associational (composition) effect was contingent on the genus (Fig. 2, Appendix B: Table B2; mixed model, composition $\times$ genus interaction, $P<0.001$ ). Specifically, the preferred goldenrods suffered up to $70 \%$ higher damage when growing in the

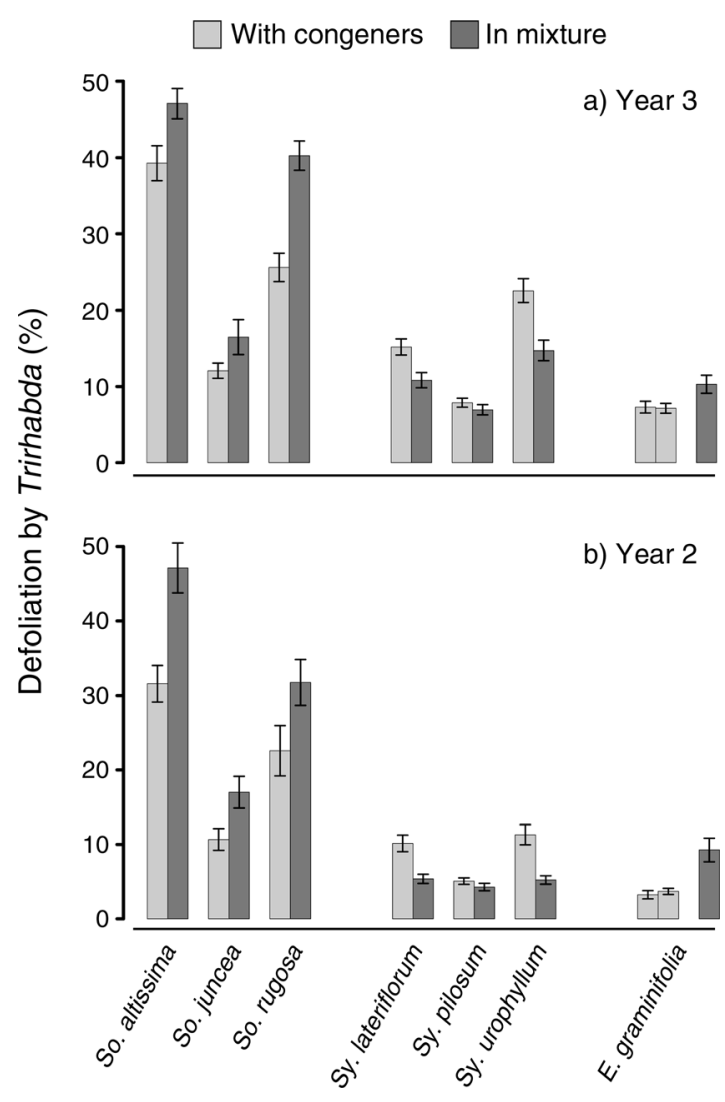

FIG. 2. By-species comparison of defoliation (percentage of leaf area consumed per ramet) by Trirhabda virgata larvae between the respective goldenrod (Solidago spp.) or aster (Symphyotrichum spp.) congeneric communities (light bars; Euthamia graminifolia present in both) and mixtures (dark bars) exposed to manipulated outbreaks in year 2 and 3 . Values are means $\pm \mathrm{SE} ; n=20$ mesocosms per treatment. 
Insect exclusion Insect herbivory
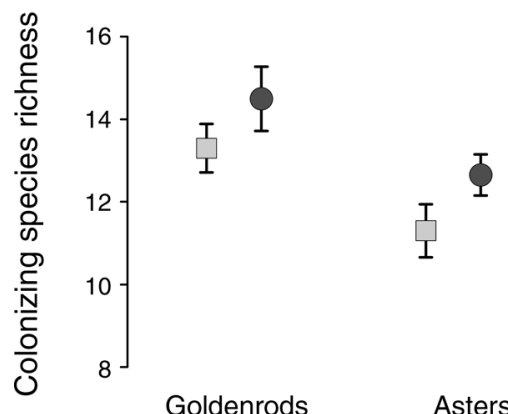

Asters
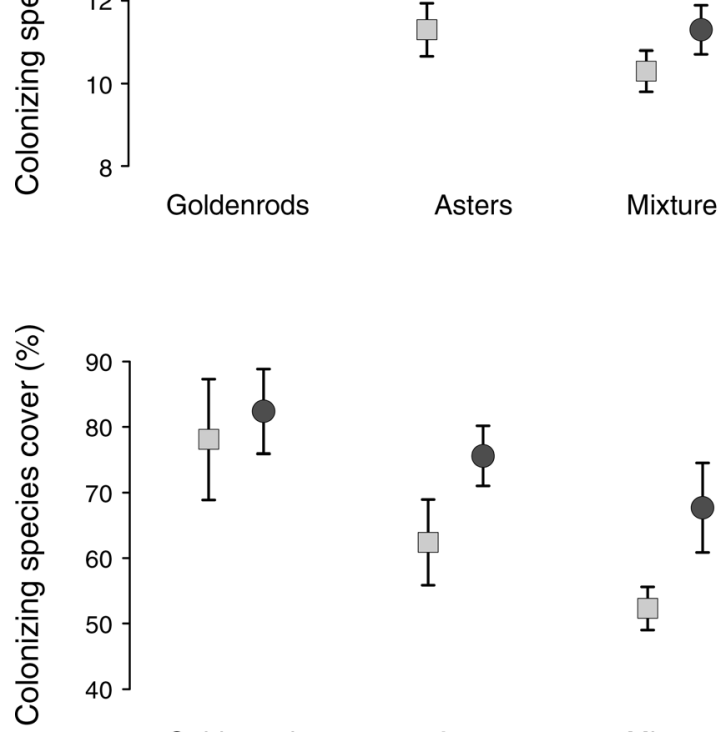

Goldenrods

Asters

Mixtures

FIG. 3. Community assembly by colonizing plant species in terms of their species richness (top) or total percent cover (bottom; allowing for overlapping vegetation, i.e., values $>100 \%$ are possible), comparing the three types of community composition (congeneric goldenrods, congeneric asters, and the mixtures of the two genera) after two years of herbivore exclusion (light squares) vs. augmented insect herbivory (dark circles). Values are means $\pm \mathrm{SE} ; n=20$ mesocosms per treatment.

mixtures than when growing with their congeners. In contrast, Symphyotrichum spp. benefited from up to a $54 \%$ reduction in damage when growing in the mixtures compared to their own congeneric communities. Therefore, the high community-level defoliation of the mixtures could be attributed to elevated herbivory on the goldenrods and reduced herbivory on the asters. Overall, neither composition nor genus were significant predictors of defoliation, but the effect of composition differed between the years (Table B2). Excluding Euthamia from the analyses yielded similar results (not shown). By the end of year 2, these patterns had been attenuated by subsequent (cumulative) insect herbivory and compensatory plant growth (Appendix A: Fig. A1, Appendix B: Table B3).

Impacts of herbivory on community structure.-Herbivory reduced community productivity (total aboveground biomass) by $15 \%$ in year 2 and by $11 \%$ in year 3 (Appendix A: Fig. A2 and Appendix B: Table B4; overall herbivory effect in mixed model, $P<0.0001$ ). However, this impact of herbivory was comparable across the composition treatments (herbivory $\times$ composition interaction not significant).

Insect herbivory influenced continued community assembly by promoting plant colonization; mesocosms with herbivory had $10 \%$ more plant species than those with insects excluded (Fig. 3, ANOVA, $F_{1,114}=4.338, P$ $=0.039$ ), and the colonizing species were also $14 \%$ more abundant (total percent cover, ANOVA, $F_{1,114}=4.934$, $P=0.028)$. Fewer species, but at similar overall abundance, colonized the mixtures than expected (contrast of mean congeneric communities and mixtures: $t=3.057, P=0.0028$ and $t=1.495, P=0.138$, respectively), and in both cases composition influenced colonizers (richness $F_{1,114}=11.62, P<0.0001$; total cover $\left.F_{1,114}=3.388, P=0.037\right)$. However, herbivory promoted colonization regardless of the initial community composition, as herbivory $\times$ composition interaction was not significant for either variable (richness $F_{2,114}=0.245, P=0.783$; total cover $F_{2,114}=0.693, P=$ 0.502). The mesocosms were colonized by 48 plant species of both native and exotic origin, spanning 33 botanical families, and, through only minor seedling recruitment, by the focal species. Most colonizers were confined to the understory of the focal species, and constituted $5-10 \%$ of the entire community biomass (based on a subsample of mesocosms).

After two years of manipulated insect herbivory, we observed shifts in the proportions of the focal species (Fig. 4), and this effect was contingent both on community composition and genus (Appendix B: Table B5; mixed model, composition $\times$ herbivory $\times$ genus interaction, $P=0.0082$ ). In other words, the composition-dependent impact of herbivory on species relative abundances differed for the two groups of host plants: although both showed more pronounced changes in their abundances under herbivory in the mixtures than in their respective congeneric communities, on average, the goldenrods tended to decrease in abundance whereas the asters tended to increase (Fig. 4). While the response of the two genera to the context of community composition also differed (composition $\times$ genus interaction, $P=0.044)$, the overall effects of composition and herbivory were not significant. Likewise, there was no overall difference between the genera, due to idiosyncratic responses of the individual species, some of which appeared to benefit indirectly from herbivory (Fig. 4). The covariate of initial relative abundances of the species prior to the herbivory treatment (i.e., in year 1) was significant (Table B5).

The influence of community composition on species shifts in abundance in response to herbivory was most conspicuous in So. altissima (Fig. 4). Although neither herbivory nor composition alone had a consistent effect on its proportional biomass across the two years of Trirhabda outbreaks (Table B6; mixed model, herbivory effect, $P=0.540$; composition effect, $P=0.09$ ), herbivory only reduced So. altissima relative abundance in the mixtures (Table B6; composition $\times$ herbivory 
interaction, $P=0.018$ ). Similarly, when the variation in the degree of herbivory (i.e., damage, in the presence of herbivory) was considered, damage (year 2 but not year 3 ) was a predictor of the final proportional biomass in the mixtures (Table B7, standardized coefficient $=0.444$ ) but not in the congeneric communities of goldenrods. In contrast, previous abundance of So. altissima (year 2) was a strong predictor of its final relative abundance in both community types (Table B7, standardized coefficients $=0.641$ and 0.676 , respectively).

\section{DisCUSSION}

Our understanding of how the variation in plant community structure drives patterns of herbivory, and how herbivores in turn shape community structure, is surprisingly incomplete, with few empirical studies having examined both phenomena simultaneously. The evidence from our three-year field experiment suggests that plant spatial structure and herbivory may reciprocally influence each other, potentially mediating plant coexistence and maintenance of fine-scale heterogeneity. Below we discuss how associational effects involving an outbreaking insect herbivore may favor higher concentrations of its preferred hosts, the goldenrods, and, conversely, may promote species evenness in patches where the less preferred hosts, the asters, can benefit from elevated herbivory on the goldenrods, suppressing their dominance.

Effects of community context on herbivory.-We present evidence that fine-scale plant community structure influenced patterns of insect herbivory among oldfield Asteraceae. Our manipulation of the initial community composition dramatically altered herbivory on the individual species by Trirhabda larvae, the pattern of which can be explained by the feeding preference hierarchy (M. Stastny and A. Agrawal, unpublished manuscript). The less-preferred asters benefited from associational resistance when intermixed with goldenrods; in contrast, the preferred goldenrods suffered from associational susceptibility, or possibly a dilution effect, in this community context, compared to when growing among their respective congeners. Although we are unable to separate the effects of resource frequency and density in our design, we interpret the lower defoliation of goldenrods in congeneric communities as an example of per-individual dilution of herbivory (e.g., Otway et al. 2005, Hambäck et al. 2014): when the preferred hosts are locally abundant, each ramet receives only moderate damage, possibly irrespective of the neighbor identity. Nonetheless, if such divergent associational (or dilution) effects in turn impact individual species abundances, herbivory and community structure may be reciprocally linked.

The mechanisms and outcomes of associational effects inherently involve both plant community characteristics and life history traits of herbivores (White and Whitham 2000, Agrawal 2004, Barbosa et al. 2009, Plath et al. 2012). Because we created Trirhabda outbreaks experi-
With congeners $\square$ In mixture

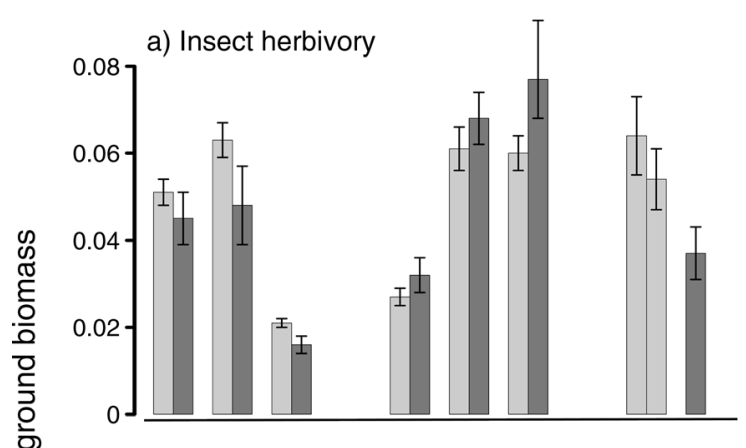

b) Insect exclusion

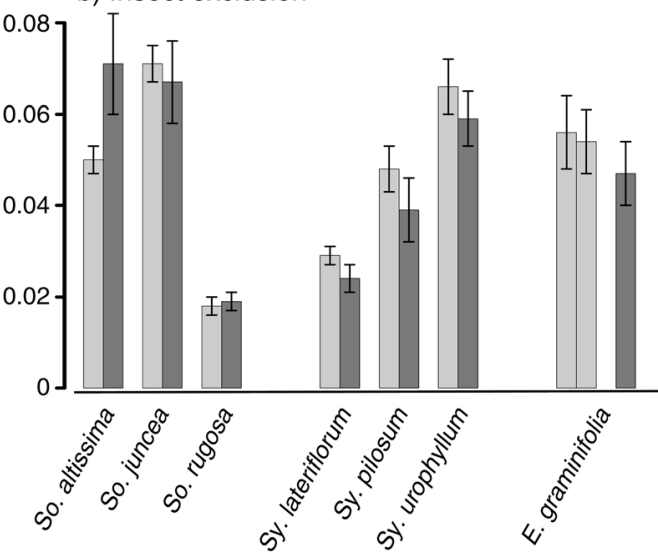

FIG. 4. Comparison of the relative abundances of individual species (per-genet proportional aboveground biomass; see Methods: Impacts of herbivory on community structure) after two years of herbivore exclusion vs. augmented insect herbivory, in their respective goldenrod (Solidago spp.) or aster (Symphyotrichum spp.) congeneric communities (light bars; $E$. graminifolia present in both) and in the mixtures of the two genera (dark bars). Bars show means $\pm \mathrm{SE} ; \mathrm{n}=20$ mesocosms per treatment.

mentally, our study removed the role of host searching (Hambäck et al. 2014), as well as factors that promote outbreaks. Consequently, the observed associational effects may not apply at larger spatial scales or reflect resource-driven distribution of this stochastically outbreaking herbivore. However, we believe that several aspects of this well-studied system support the observed patterns. All of the hosts co-occur naturally at the level of microsites. This vegetation heterogeneity may be crucial, as Trirhabda lays overwintering eggs on the soil and detritus (rather than on its hosts). The relatively mobile larvae then search for hosts, and continue to move among them frequently, facilitating differential feeding that reflects host preference. In patches with greater species evenness, defoliation of goldenrods may be near $100 \%$ even at intermediate per-unit-area Trirhabda densities (M. Stastny, personal observation) that could reflect lower oviposition. In contrast, nearmonocultures of the clonal goldenrods (especially So. altissima) often escape heavy defoliation through 
numeric dilution of damage, except in extreme outbreaks (Carson and Root 2000). Yet, such host concentrations may be creating outbreak dynamics in the first place, and driving spatiotemporal distribution of the herbivores (Herzig and Root 1996, Long et al. 2003, Carson et al. 2004, Stephens and Myers 2012). In a complex interplay of herbivore dynamics and vegetation heterogeneity, associational effects may thus lead to suppression of the preferred hosts in mixed patches, thereby promoting plant coexistence, and, conversely, favoring their spatial concentration.

Impacts of herbivory on community structure.-The most obvious top-down effect of herbivores on plant communities is via the removal of primary productivity (Crawley 1989). In our study, insect herbivory reduced the biomass of the focal goldenrods and asters by 1115\% (Appendix A: Fig. A2), an amount comparable to the mean levels reported for grassland and herbaceous communities (Coupe and Cahill 2003). However, because biomass is consumed differentially, and species vary in their tolerance of damage (Trumble et al. 1993), herbivores may also alter plant competitive interactions and influence ongoing community assembly (Uriarte 2000, Bach 2001, Crawley 2009). Therefore, we examined whether insect herbivory and its variation due to the associational effects subsequently elicited changes in community structure.

First, insect herbivory promoted colonization of the mesocosms by other plant species (Fig. 3), in agreement with previous studies in this system in which plant richness or abundance increased following bouts of insect herbivory (e.g., Carson and Root 2000). The impacts of herbivory on community assembly did not vary with community composition, potentially because the three community types reached similar biomass under herbivore exclusion, and suffered comparable total biomass reductions due to herbivory. The facilitation of colonization through herbivory may have occurred through at least two distinct mechanisms. First, opportunities for colonization and establishment may have improved when the focal species were suppressed by Trirhabda outbreaks early in the season. Such effects of herbivores on the release of competitively inferior species are well documented, and can translate to long-term impacts on community structure (Crawley 1989, Bach 2001, Hillebrand et al. 2007). Second, the colonizing species themselves were also subject to a variety of herbivores (not measured), which may have affected their persistence and competitive interactions (Agrawal et al. 2012). Via these indirect mechanisms, insect herbivores may thus shape community assembly and promote local plant diversity in old fields.

Second, we show that associational effects likely contributed to subsequent changes in the relative abundance of the focal goldenrods and asters. Not only was insect herbivory associated with changes in the proportional biomass of some of the individual species (Fig. 4), but, crucially, the overall shift in the focal community structure over two years of outbreaks depended on the initial community composition. While these effects were contingent on the plant genus (i.e., significant composition $\times$ herbivory $\times$ genus interaction), since goldenrods and asters showed contrasting associational effects, they suggest that variation in herbivory may contribute to changes in plant community structure. Our study did not separate the influence of associational effects on shifts in community structure from those by plant competition, which can also be expected to depend on neighbor identity or community composition (Underwood et al. 2014).

For the individual species, the difference in damage between the composition treatments was generally a poor predictor of the difference in their relative abundance (see Figs. 2 and 4), which may be unsurprising given the complexity of community interactions, including interspecific plant competition (Cronin et al. 2010). Plant species also likely markedly differed in tolerance and some were able to regrow substantially after Trirhabda outbreaks, in spite of continued (albeit less intense) herbivore pressure (see results on cumulative damage; Appendix B). The clonal goldenrods, in particular, possess a remarkable ability to tolerate herbivory (Root 1996, Carson and Root 2000), mediated by their belowground allocation that facilitates compensatory growth (Root and Cappuccino 1992, Uriarte 2000). A long-term study by Carson and Root (2000) observed an eventual reduction in the dominance of So. altissima only following several years of outbreaks by another, more strictly specialized, Chrysomelid, Microrhopala vittata. In spite of the shorter duration of our study, our findings hint at the potential role of associational effects of herbivory in shaping the patterns and dynamics of fine-scale plant community heterogeneity.

The most pronounced impact of herbivory in our study was on So. altissima, the preferred host of Trirhabda. While herbivory did not alter its relative abundance in congeneric (goldenrod) communities, it reduced it by $37 \%$ in the mixtures (Fig. 4), where So. altissima experienced higher damage. Similarly, only in the mixtures was Trirhabda damage a significant predictor of the abundance of So. altissima in the final year. Therefore, divergent associational effects experienced by So. altissima in the two types of communities were reflected in the corresponding changes in its hierarchy in the community, although we again note that we did not separate the relative contribution of associational (or dilution) effects from that of the changing competition due to neighbor identity. Our findings imply that responses of So. altissima may explain a substantial portion of the observed overall shift in community structure due to herbivory. As this species tends to be a competitive dominant in old-field communities in the region, its fine-scale suppression by specialist herbivores may be paramount in promoting local native plant diversity (Stastny 2010). 
Interplay of community structure and herbivory.Researchers have long been interested in identifying ecological contexts in which herbivores have strong topdown effects on plant communities (Carson et al. 2004, Cronin et al. 2010, Stein et al. 2010, Olofsson et al. 2013). The idea of feedbacks between plant community structure and herbivory adds another dimension to this search for a predictive framework. The substitutive (replacement) design of our experiment precludes direct tests of such feedbacks, and a longer time scale would likely be required. We have outlined the individual components of the reciprocal effects of community structure on herbivory, and vice versa, within the same experimental study-necessary but not sufficient to demonstrate feedbacks. One interesting implication of our study is that, contrary to the positive relationship between resource concentration and herbivory suggested by some mechanistic models of herbivore impacts on communities (e.g., Carson et al. 2004), the decreasing abundance of a preferred species at the neighborhood scale may elevate its herbivory and further reduce its abundance. Top-down effects of outbreaking insects that utilize multiple co-occurring hosts may thus simultaneously favor dense monospecific (or congeneric) stands of the preferred host(s) via dilution of herbivory, while maintaining its low abundance in diverse patches where the less-preferred hosts escape herbivory and benefit from competitive release. Finally, as populations of plants and herbivores continue to vary in time and space, feedbacks between local plant community structure and herbivory are likely to be dynamic and may give rise to a complex interplay of directional vs. stabilizing effects in the local community (Vellend 2010).

\section{ACKNOWLEDGMENTS}

For the assistance with the experimental setup, data collection, or analysis, we thank N. Becker, S. Campbell, S. McArt, J. Koslow, J. Lamb, J. Parker, K. Mooney, J. Simonis, S. Cook-Patton, A. Erwin, Cornell Farm Services, and especially M. Glaymour. Earlier versions of this paper benefited from comments by M. Geber and her lab group, A. Kessler, R. B. Root, the Agrawal lab, J. Parker, S. McArt, T. Pendergast, P. Hambäck, and three anonymous reviewers. Funding was provided by Cornell's Biogeochemistry and Environmental Biocomplexity Small Grant (DGE 0221658), Andrew W. Mellon Student Research Grant, and NSF-DEB 1118783 (A. A. Agrawal). M. Stastny was supported by the Postgraduate Scholarship from the National Science and Engineering Research Council (NSERC) of Canada during the course of this study.

\section{Literature Cited}

Agrawal, A. A. 2004. Resistance and susceptibility of milkweed: competition, root herbivory, and plant genetic variation. Ecology 85:2118-2133.

Agrawal, A. A., A. P. Hastings, M. T. Johnson, J. L. Maron, and J.-P. Salminen. 2012. Insect herbivores drive real-time ecological and evolutionary change in plant populations. Science 338:113-116.

Agrawal, A. A., J. A. Lau, and P. A. Hambäck. 2006. Community heterogeneity and the evolution of interactions between plans and insect herbivores. Quarterly Review of Biology 81:349-376.
Atsatt, P. R., and D. J. O'Dowd. 1976. Plant defense guilds. Science 193:24-29.

Bach, C. E. 2001. Long-term effects of insect herbivory and sand accretion on plant succession on sand dunes. Ecology 82:1401-1416.

Barbosa, P., J. Hines, I. Kaplan, H. Martinson, A. Szczepaniec, and Z. Szendrei. 2009. Associational resistance and susceptibility: having right or wrong neighbors. Annual Review of Ecology, Evolution, and Systematics 40:1-20.

Bolker, B. M. 2008. Ecological models and data in R. Princeton University Press, Princeton, New Jersey, USA.

Brown, V. K., and A. C. Gange. 1992. Secondary plant succession: how is it modified by herbivory? Functional Ecology 3:667-671.

Carson, W. P., J. P. Cronin, and Z. T. Long. 2004. A general rule for predicting when insects will have strong top-down effects on plant communities: on the relationship between insect outbreaks and host concentration. Pages 193-211 in W. W. Weisser and E. Siemann, editors. Insects and ecosystem function. Springer-Verlag, Berlin, Germany.

Carson, W. P., and R. B. Root. 2000. Herbivory and plant species coexistence: community regulation by an outbreaking phytophagous insect. Ecological Monographs 70:73-99.

Coupe, M. D., and J. F. Cahill. 2003. Effects of insects on primary production in temperate herbaceous communities: a meta-analysis. Ecological Entomology 28:511-521.

Crawley, M. J. 1989. Insect herbivores and plant population dynamics. Annual Review of Entomology 34:531-564.

Crawley, M. J. 2002. Statistical computing: an introduction to data analysis using S-PLUS. John Wiley and Sons, London, UK.

Crawley, M. J. 2009. Plant-herbivore dynamics. Pages 475-531 in M. J. Crawley, editor. Plant ecology. Blackwell Science, London, UK.

Cronin, J. P., S. J. Tonsor, and W. P. Carson. 2010. A simultaneous test of trophic interaction models: which vegetation characteristic explains herbivore control over plant community mass? Ecology Letters 13:202-212.

Daleo, P., B. Silliman, J. Alberti, M. Escapa, N. Peña, and O. Iribarne. 2009. Grazer facilitation of fungal infection and the control of plant growth in south-western Atlantic salt marshes. Journal of Ecology 97:781-787.

Doak, P. 2000. Habitat patchiness and the distribution, abundance, and population dynamics of an insect herbivore. Ecology 81:1842-1857.

Feeny, P. P. 1976. Plant apparency and chemical defense. Pages 1-40 in J. W. Wallace and R. L. Mansell, editors. Biochemical interaction between plants and insects. Plennum, New York, New York, USA.

Fraenkel, G. 1959. The raison d'etre of secondary plant substances. Science 129:1466-1470.

Fritz, R. S., and E. L. Simms. 1992. Plant resistance to herbivores and pathogens: ecology, evolution and genetics. University of Chicago Press, Chicago, Illinois, USA.

Futuyma, D. J., and A. A. Agrawal. 2009. Macroevolution and the biological diversity of plants and herbivores. Proceedings of the National Academy of Sciences USA 106:18054-18061.

Grover, J. P. 1994. Assembly rules for communities of nutrientlimited plants and specialist herbivores. American Naturalist 143:258-282.

Hambäck, P. A., J. Agren, and L. Ericson. 2000. Associational resistance: insect damage to purple loosestrife reduced in thickets of sweet gale. Ecology 81:1784-1794.

Hambäck, P. A., and A. P. Beckerman. 2003. Herbivory and plant resource competition: a review of two interacting interactions. Oikos 101:26-37.

Hambäck, P. A., B. D. Inouye, P. Andersson, and N. Underwood. 2014. Effects of plant neighbourhoods on plant-herbivore interactions: resource dilution and associational effects. Ecology 95:1370-1383. 
Herzig, A. L. 1995. Effects of population density on longdistance dispersal in the goldenrod beetle Trirhabda virgata. Ecology 76:2044-2054.

Herzig, A. L., and R. B. Root. 1996. Colonization of host patches following long-distance dispersal by a goldenrod beetle, Trirhabda virgate. Ecological Entomology 21:344351.

Hillebrand, H., et al. 2007. Consumer versus resource control of producer diversity depends on ecosystem type and producer community structure. Proceedings of the National Academy of Sciences USA 104:10904-10909.

Karban, R. 1997. Neighborhood affects a plant's risk of herbivory and subsequent success. Ecological Entomology 22:433-439.

Karban, R., and I. T. Baldwin. 1997. Induced responses to herbivory. University of Chicago Press, Chicago, Illinois, USA.

Long, Z. T., C. L. Mohler, and W. P. Carson. 2003. Extending the resource concentration hypothesis to plant communities: effects of litter and herbivores. Ecology 84:652-665.

Maddox, G. D., and R. B. Root. 1990. Structure of the encounter between goldenrod (Solidago altissima) and its diverse insect fauna. Ecology 71:2115-2124.

Mitchell, C. E. 2003. Trophic control of grassland production and biomass by pathogens. Ecology Letters 6:147-155.

Ness, J. H., E. J. Rollinson, and K. D. Whitney. 2011. Phylogenetic distance can predict susceptibility to attack by natural enemies. Oikos 120:1327-1334.

Olofsson, J., M. te Beest, and L. Ericson. 2013. Complex biotic interactions drive long-term vegetation dynamics in a subarctic ecosystem. Philosophical Transactions of the Royal Society B 368:20120486.

Otway, S. J., A. Hector, and J. H. Lawton. 2005. Resource dilution effects on specialist insect herbivores in a grassland biodiversity experiment. Journal of Animal Ecology 74:234240.

Parker, M., and R. B. Root. 1981. Insect herbivores limit habitat distribution of a native composite, Machaeranthera canescens. Ecology 62:1390-1392.

Pearse, I. S., and A. L. Hipp. 2009. Phylogenetic and trait similarity to a native species predict herbivory on non-native oaks. Proceedings of the National Academy of Sciences USA 106:18097-18102.

Plath, M., S. Dorn, J. Riedel, H. Barrios, and K. Mody. 2012. Associational resistance and associational susceptibility: specialist herbivores show contrasting responses to tree stand diversification. Oecologia 169:477-487.
R Development Core Team. 2013. R version 2.15.2. R Project for Statistical Computing, Vienna, Austria. www.r-project. org

Root, R. B. 1996. Herbivore pressure on goldenrods (Solidago altissima): its variation and cumulative effects. Ecology 77 : 1074-1087.

Root, R. B., and N. Cappuccino. 1992. Patterns in population change and the organization of the insect community associated with goldenrod. Ecological Monographs 63:393420.

Schadler, M., G. Jung, R. Brandl, and H. Auge. 2004. Secondary succession is influenced by belowground insect herbivory on a productive site. Oecologia 138:242-252.

Schmitz, O. J. 2008. Herbivory from individuals to ecosystems. Annual Review of Ecology, Evolution, and Systematics 39: $133-152$.

Stastny, M. 2010. Ecological consequences of relatedness: the role of competition and herbivory in the community structure of co-occurring Asteraceae. Dissertation. Department of Ecology and Evolutionary Biology, Cornell University, Ithaca, New York, USA.

Stein, C., S. B. Unsicker, A. Kahmen, M. Wagner, V. Audorff, H. Auge, D. Prati, and W. W. Weisser. 2010. Impact of invertebrate herbivory in grasslands depends on plant species diversity. Ecology 91:1639-1650.

Stephens, A. E. A., and J. H. Myers. 2012. Resource concentration by insects and implications for plant populations. Journal of Ecology 100:923-931.

Trumble, J. T., D. M. Kolodny-Hirsch, and I. P. Ting. 1993. Plant compensation for arthropod herbivory. Annual Review of Entomology 38:93-119.

Underwood, N., and S. Halpern. 2012. Insect herbivores, density dependence, and the performance of the perennial herb Solanum carolinense. Ecology 93:1026-1035.

Underwood, N., B. D. Inouye, and P. A. Hambäck. 2014. A conceptual framework for associational effects: when do neighbours matter and how would we know? Quarterly Review of Biology 89:1-19.

Uriarte, M. 2000. Interactions between goldenrod (Solidago altissima L.) and its insect herbivore (Trirhabda virgata) over the course of succession. Oecologia 122:521-528.

Vellend, M. 2010. Conceptual synthesis in community ecology. Quarterly Review of Biology 85:183-206.

White, J. A., and T. G. Whitham. 2000. Associational susceptibility of cottonwood to a box elder herbivore. Ecology 81:1795-1803.

\section{Supplemental Materials}

\section{Appendix A}

Plot of cumulative (end-of-season, year 2) insect herbivory by composition treatment and plot of community productivity (total mesocosm biomass) across composition and herbivory treatments and years (Ecological Archives E095-251-A1).

\section{Appendix B}

Summary tables of statistical analyses of the effects of community context on herbivory and of the impacts of herbivory on community structure (Ecological Archives E095-251-A2). 


\section{Ecological Archives E095-251-A1}

\section{Michael Stastny and Anurag A. Agrawal. 2014. Love thy neighbor? reciprocal impacts between plant community structure and insect herbivory in co-occurring Asteraceae. Ecology 95:2904-2914. http://dx.doi.org/10.1890/13-1115.1}

ApPENDix A. Plot of cumulative (end-of-season, year 2) insect herbivory by composition treatment and plot of community productivity (total mesocosm biomass) across composition and herbivory treatments and years.

\section{Cumulative percent defoliation}

So. altissima

So. juncea

So. rugosa

Sy. lateriflorum

Sy. pilosum

Sy. urophyllum

E. graminifolia

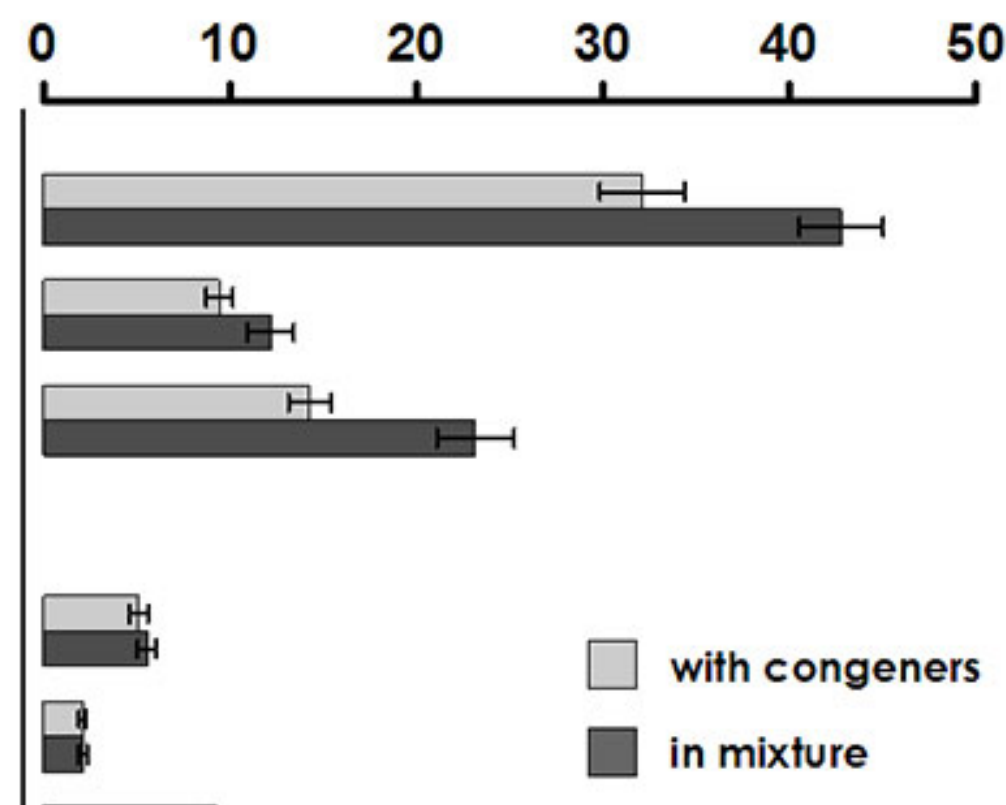

FIG. A1. By-species comparison of cumulative (end-of-season) insect herbivory in year 2 (in the presence of insect herbivory), measured as percent leaf area consumed per ramet, between their respective congeneric communities and mixtures. This relative metric integrates Trirhabda damage, feeding by a variety of other insect herbivores, and plant growth/regrowth. Means \pm standard errors; $n=20$ per 


\section{$\square$ insect exclusion $\bigcirc$ insect herbivory}

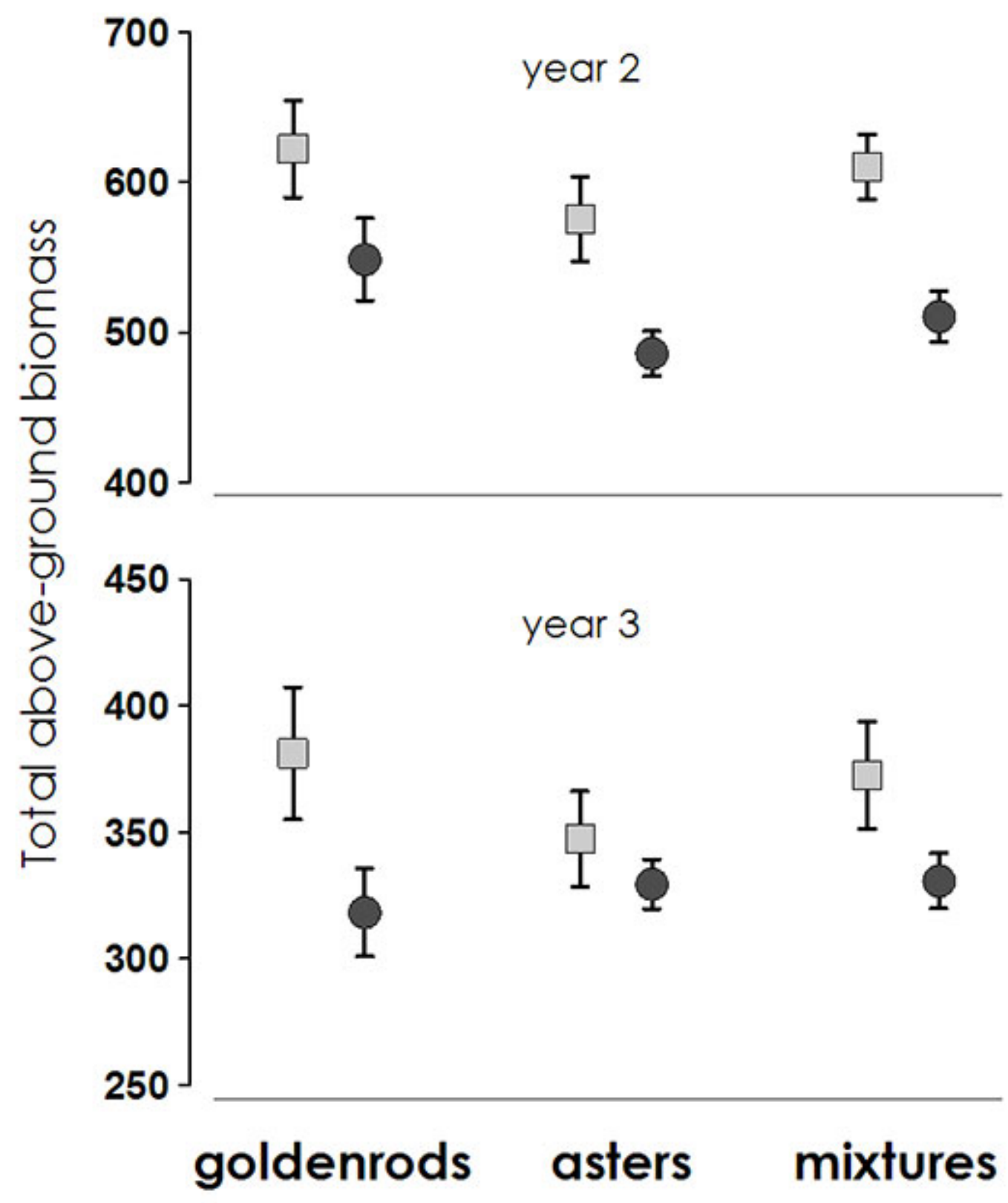

Fig. A2. Comparison of total community productivity, measured as the above-ground biomass (grams of dry biomass), in the three composition treatments - congeneric community of goldenrods, congeneric 
community of asters, and the mixture of the genera - under insect herbivore exclusion vs. manipulated insect herbivory, in year 1 (top) and year 2 (bottom). Means \pm standard errors; $n=20$ per treatment. 


\section{Ecological Archives E095-251-A2}

\section{Michael Stastny and Anurag A. Agrawal. 2014. Love thy neighbor? reciprocal impacts between plant community structure and insect herbivory in co-occurring Asteraceae. Ecology 95:2904-2914. http://dx.doi.org/10.1890/13-1115.1}

APPENDix B. Summary tables of statistical analyses of the effects of community context on herbivory and of the impacts of herbivory on community structure.

TABLE B1. Summary of the repeated-measures linear mixed model (function lmer in R) on community-level Trirhabda herbivory in each year of manipulated outbreaks (years 2 and 3), with the average per-ramet defoliation (percent leaf area removed) as the response variable. Composition 1 represents the contrast between congeneric goldenrod and congeneric aster communities; composition 2 represents the contrast between the mean congeneric communities (of goldenrods and asters combined) and mixtures; see text for details. Mesocosm was included as a random effect $(n=60)$. MCMC sampling (function pvals.fnc) was used to estimate $p$ values for the fixed effects.

\begin{tabular}{|l|r|r|r|r|l|}
\hline \multicolumn{1}{|c|}{ Fixed effects } & Estimate & Std. Error & $\boldsymbol{t}$ value & $\operatorname{Pr}(>|\mathbf{t}| \mathbf{)}$ & \\
\hline (Intercept) & 2.216 & 0.084 & 26.512 & $<0.001$ & $* * *$ \\
\hline composition1 & 0.442 & 0.102 & 4.318 & $<0.001$ & $* * *$ \\
\hline composition2 & -0.140 & 0.059 & -2.375 & 0.019 & $*$ \\
\hline year & 0.330 & 0.052 & -2.375 & $<0.001$ & $* * *$ \\
\hline composition1:year & -0.119 & 0.064 & -1.855 & 0.072 & \\
\hline composition2:year & 0.038 & 0.037 & -1.033 & 0.304 & \\
\hline
\end{tabular}

TABLE B2. Summary of the repeated-measures linear mixed model (function lmer in R) on Trirhabda herbivory at the species level in each year of manipulated outbreaks (years 2 and 3), with the average perramet defoliation (percent leaf area removed) as the response variable. Composition has two levels (congeneric, mixtures) in this analysis; genus also has two levels (goldenrods, including E. graminifolia, and asters). Composition1 represents the contrast between congeneric goldenrod and congeneric aster communities; composition2 represents the contrast between the mean congeneric communities (of goldenrods and asters combined) and mixtures; see text for details. Species ( 7 levels $)$ and mesocosm $(n=$ 
60) were included as random effects. MCMC sampling (function pvals.fnc) was used to estimate $p$ values for the fixed effects.

\begin{tabular}{|l|r|r|r|r|r|}
\hline \multicolumn{1}{|c|}{ Fixed effects } & Estimate & Std. Error & $\boldsymbol{t}$ value & $\operatorname{Pr}(>|\mathbf{t}|)$ & \\
\hline (Intercept) & 3.473 & 0.333 & 10.419 & $<0.001$ & $* * *$ \\
\hline composition & 0.120 & 0.119 & 1.018 & 0.309 & \\
\hline genus & -0.609 & 0.499 & -1.222 & 0.222 & \\
\hline year & 0.330 & 0.052 & -2.375 & $<0.001$ & $* * *$ \\
\hline composition:genus & -0.299 & 0.076 & -3.390 & $<0.001$ & $* * *$ \\
\hline composition1:year & -0.368 & 0.042 & -8.807 & $<0.001$ & $* * *$ \\
\hline composition2:year & -0.394 & 0.045 & -8.821 & $<0.001$ & $* * *$ \\
\hline
\end{tabular}

TABLE B3. Summary of the linear mixed model (function lmer in R) for cumulative, end-of-season herbivory in year 2 (in the presence of insect herbivory), with the average per-ramet defoliation (percent leaf area removed) as the response variable. Species and mesocosm were included as random effects. MCMC sampling (function pvals.fnc) was used to estimate $p$ values for the fixed effects. Cumulative foliar damage was $13 \%$ higher, overall, in the mixtures than in the congeneric communities; see Fig. A1 for species-level comparisons.

\begin{tabular}{|l|r|r|r|r|r|}
\hline \multicolumn{1}{|c|}{ Fixed effects } & Estimate & Std. Error & $\boldsymbol{t}$ value & $\operatorname{Pr}(>|\mathbf{t}| \mathbf{)}$ & \\
\hline (Intercept) & 2.385 & 0.448 & 5.324 & $<0.001$ & $* * *$ \\
\hline composition & 0.206 & 0.093 & 2.221 & 0.027 & $*$ \\
\hline genus & -0.897 & 0.683 & -1.314 & 0.190 & \\
\hline
\end{tabular}


TABLE B4. Summary of the repeated-measures linear mixed model (function lmer in R) on community productivity (above-ground biomass) under manipulated Trirhabda outbreaks (years 2 and 3) vs. herbivoreexclusion treatment. Composition1 represents the contrast between congeneric goldenrod and congeneric aster communities; composition 2 represents the contrast between the mean congeneric communities (of goldenrods and asters combined) and mixtures; see text for details. Mesocosm was included as a random effect $(n=120)$. MCMC sampling (function pvals.fnc) was used to estimate $p$ values for the fixed effects.

\begin{tabular}{|l|r|r|r|r|r|}
\hline \multicolumn{1}{|c|}{ Fixed effects } & Estimate & Std. Error & $\boldsymbol{t}$ value & $\operatorname{Pr}(>|\mathbf{t}|)$ & \\
\hline Intercept) & 942.71 & 25.28 & 37.29 & $<0.001$ & $* * *$ \\
\hline composition1 & -15.56 & 13.74 & -1.13 & 0.258 & \\
\hline composition2 & -1.873 & 7.93 & -0.24 & 0.814 & \\
\hline herbivory & 67.87 & 15.86 & 4.28 & $<0.001$ & $* * *$ \\
\hline year & -210.3 & 9.06 & -23.21 & $<0.001$ & $* * *$ \\
\hline composition1:herbivory & -4.603 & 19.43 & -0.24 & 0.813 & \\
\hline composition2:herbivory & -1.382 & 11.22 & -0.12 & 0.902 & \\
\hline
\end{tabular}

TABLE B5. Summary of the linear mixed model (function lmer in R) on relative abundances of the species in the mesocosms in the final year. Proportional above-ground biomass in year 3 (biomass standardized per initial number of genets, then divided by the total community biomass) is the response variable; the model includes initial standardized biomass of the species after year 1 (biomassyear1) as a covariate. Composition has two levels (congeneric, mixtures) in this analysis; genus also has two levels (goldenrods, including $E$. graminifolia, and asters); see text for details. Species (7 levels) and mesocosm $(n=120)$ were included as random effects. MCMC sampling (function pvals.fnc) was used to estimate $p$ values for the fixed effects.

\begin{tabular}{|c|c|c|c|c|}
\hline Fixed effects & Estimate & Std. Error & $t$ value & $\operatorname{Pr}(>|\mathbf{t}|)$ \\
\hline
\end{tabular}




\begin{tabular}{|c|c|c|c|c|c|}
\hline (Intercept) & -4.074 & 0.253 & -16.131 & $<0.001$ & $* * *$ \\
\hline biomassyear1 (covariate) & 0.329 & 0.079 & 4.139 & $<0.001$ & $* * *$ \\
\hline composition & -0.152 & 0.101 & -1.498 & 0.135 & \\
\hline herbivory & 0.018 & 0.095 & 0.189 & 0.850 & \\
\hline genus & -0.141 & 0.243 & -0.580 & 0.562 & \\
\hline composition:herbivory & 0.168 & 0.142 & 1.187 & 0.236 & \\
\hline composition:genus & 0.323 & 0.160 & 2.019 & 0.044 & $*$ \\
\hline herbivory:genus & -0.057 & 0.154 & -0.368 & 0.713 & \\
\hline compos:herbivory:genus & -0.593 & 0.224 & -2.651 & 0.008 & $* *$ \\
\hline
\end{tabular}

TABLE B6. Summary of the repeated-measures linear model (function lmer in R) on the relative abundance of Solidago altissima in mesocosm communities across years 2 and 3 , under different community (composition: congeneric goldenrods, mixtures) and herbivory (excluded, manipulated outbreaks) treatments. Proportional above-ground biomass (biomass standardized per initial number of genets, then divided by the total community biomass) is the response variable; the model includes community productivity (total above-ground biomass) as a covariate, and the random effects of mesocosm $(n=80)$.

\begin{tabular}{|l|r|r|r|r|r|}
\hline \multicolumn{1}{|c|}{ Fixed effects } & Estimate & Std. Error & $\boldsymbol{t}$ value & $\operatorname{Pr}(>|\mathbf{t}|)$ & \\
\hline (Intercept) & -3.708 & 0.350 & -8.803 & $<0.001$ & $* * *$ \\
\hline productivity (covariate) & -0.001 & 0.001 & -4.054 & $<0.001$ & $* * *$ \\
\hline composition & -0.185 & 0.109 & -1.694 & 0.092 & \\
\hline
\end{tabular}




\begin{tabular}{|l|r|r|r|r|r|} 
herbivory & 0.068 & 0.111 & 0.614 & 0.540 & \\
\hline year & 0.163 & 0.084 & 1.954 & 0.054 & \\
\hline composition:herbivory & 0.367 & 0.153 & 2.395 & 0.018 & $*$ \\
\hline
\end{tabular}

TABLE B7. Summary of multiple regressions on relative abundance (proportional biomass) of Solidago altissima in year 3 when growing (a) with congeneric goldenrods, (b) in the mixtures. Proportional biomass in year 2, and feeding damage by Trirhabda in years 2 and 3, are used as predictor variables in both models. All variables have been standardized prior to analysis.

a) with goldenrods

\begin{tabular}{|l|r|r|r|r|r|}
\hline & Std. Estim. & Std. Error & $\boldsymbol{t}$ value & $\operatorname{Pr}(>|\mathbf{t}|)$ & \\
\hline (Intercept) & -0.0001 & 0.0242 & -0.790 & 0.441 & \\
\hline damage year2 & 0.211 & 0.0004 & 1.137 & 0.272 & \\
\hline prop. biomassyear2 & 0.641 & 0.442 & 3.519 & 0.003 & $* *$ \\
\hline damageyear3 & 0.099 & 0.0004 & 0.529 & 0.604 & \\
\hline
\end{tabular}

Resid. SE $(\mathrm{df}=16)=0.0173 ;$ mult. $R^{2}=0.492$, adj. $R^{2}=0.397 ; F_{3,16}=5.168, p$ value $=0.011$ b) in mixtures

\begin{tabular}{|l|r|r|r|r|r|}
\hline & Std. Estim. & Std. Error & $\boldsymbol{t}$ value & $\operatorname{Pr}(>|\mathbf{t}|)$ & \\
\hline (Intercept) & -0.0001 & 0.0239 & -0.603 & 0.555 & \\
\hline damage year2 & 0.445 & 0.0002 & 3.516 & 0.003 & $* *$ \\
\hline prop. biomassyear2 & 0.676 & 0.336 & 5.054 & $<0.001$ & $* * *$ \\
\hline
\end{tabular}




\begin{tabular}{|c|c|c|c|c|}
\hline damageyear3 & -0.200 & 0.0004 & -1.496 & 0.154 \\
\hline
\end{tabular}

Resid. SE $(\mathrm{df}=16)=0.0137$; mult. $R^{2}=0.746$, adj. $R^{2}=0.698 ; F_{3,16}=15.63, p$ value $<0.001$ 